\title{
The eigenfunction expansion of the infinite depth free surface Green function in three dimensions
}

\author{
Malte A. Peter ${ }^{a, *}$ Michael H. Meylan ${ }^{\mathrm{a}, * *}$ \\ ${ }^{a}$ Institute of Information and Mathematical Sciences, Massey University, \\ Auckland, New Zealand
}

\begin{abstract}
A new representation of the infinite depth free surface Green function in three dimensions is derived. This representation is in the eigenfunction expansion of an outgoing wave centred at the source point of the Green function. Such a representation allows the calculation of the scattered potential in terms of the eigenfunctions of an outgoing wave. Furthermore this new representation of the Green function is found to compare favourably with existing representations in terms of its naive numerical evaluation. We also show that the eigenfunction representation can be retained in a new coordinate system which is not centred at the source point of the Green function.
\end{abstract}

Key words: Free surface Green function; water waves; eigenfunction expansion

\section{Introduction}

The scattering of water waves by a floating or submerged body or the radiation of water waves by an oscillating structure are common problems to be solved in ocean engineering. If the wave amplitudes are sufficiently small the solution may be approximated by that of the linearised problem. The standard and most general solution method for this linear boundary value problem is to transform it into an integral equation over the wetted body surface. The wetted

* Present address: Zentrum für Technomathematik, Universität Bremen, Germany ** Present address: Department of Mathematics, University of Auckland, New Zealand

Email addresses: mpeter@math.uni-bremen.de (Malte A. Peter), meylan@math. auckland.ac.nz (Michael H. Meylan). 
body surface is then discretised and the solution of the scattering or radiation problem is found numerically. This transformation to an integral equation requires a special Green function known as the free surface Green function.

Free surface Green functions have been known since the work of John [7] in the middle of the last century. John derived a variety of representations of the Green function in two and three dimensions but the work is best known for a representation of the Green function for three-dimensional water of constant finite depth. John expressed this Green function in the eigenfunctions of an outgoing wave centred at the source point. While the finite depth Green function can be used for all applications, it is well known that if the water is sufficiently deep (which is often the case in practical applications) the water depth may be approximated as infinite. This approximation has the advantage of using Green functions which can be computed more rapidly.

While John [7] derived a Green function for infinite depth the first practical representation was given by Havelock [5]. However, Havelock's representation was still numerically difficult because it required the integration of a singular function. Based on Havelock's work, Kim [9] was able to derive a representation for the Green function in three dimensions for water of infinite depth which only required the integration of a non-singular integrand. However, Kim's representation included an uncommon special function, the Struve function, which does not appear in most major commercial software products. Furthermore, for certain parameters, the integrand in Kim's representation is very steep which complicates its numerical integration. None of these representations expressed the three-dimensional infinite depth Green function as an expansion in the eigenfunctions of an outgoing wave.

There has also been significant research on developing methods to very efficiently evaluate the free surface Green functions numerically, lead by the demands of commercial software. Significant research in this area has been performed by Newman [11] and Noblesse and co-workers [12,13]. Newman's method to evaluate the Green function was based on tables of approximating polynomials while Noblesse's method was based on using different expansions in different regions. To the best of the authors' knowledge no paper has appeared in which the methods of Noblesse and Newman are compared. It should also be noted that these methods, while quick to evaluate the Green function, would be slow and difficult to implement, and hence be unsuitable for many applications.

In some applications it is important that the scattered potential is given in the eigenfunction expansion for outgoing waves, sometimes even in a fixed coordinate system which is not centred at the source point. The calculation of diffraction transfer matrices for bodies of arbitrary shape in Kagemoto 85 Yue's interaction theory [8], for example, requires the representation of the Green function in the eigenfunction expansion where the coordinate system is fixed at 
the mean centre position of the body. In finite depth, such a Green function was derived by Black [2] and Fenton [3] based on John's representation of the Green function in the eigenfunction expansion. This Green function has been used to calculate the diffraction transfer matrices for bodies of arbitrary geometry by Goo $\mathscr{E}$ Yoshida [4]. In water of infinite depth, however, the lack of an eigenfunction expansion of the Green function prohibited such calculations.

In this paper, the eigenfunction expansion of the infinite depth free surface Green function in three dimensions is derived from the equivalent finite depth Green function which was given by John. This new representation of the infinite depth Green function, which is easy to evaluate because it only involves common special functions and the integration of a non-singular integrand, is compared numerically to some other known representations. This comparison is for a naive implementation and does not consider any of the acceleration methods of Newman or Noblesse. It is also illustrated how the eigenfunction representation of the infinite depth Green function can be obtained in a coordinate system which is not centred at the source point.

\section{The Green function}

The standard solution method for the linear free surface water wave scattering or radiation is to transform the problem into an integral equation using a Green function. The integral equation, which is restricted to the immersed body surface, is then solved. The Green function used in this method must satisfy the free surface, sea floor, and radiation conditions as well as its Laplacian being a delta function. This boundary value problem for the Green function and the associated integral equation is briefly presented.

Let $\omega$ denote the radian frequency of the ambient wave and $\alpha=\omega^{2} / g$ where $g$ is the acceleration due to gravity. For simplification, $\mathbf{x}=(x, y, z)$ and $\xi=$ $(a, b, c)$ always represent points in the water. Let $D$ denote the lower threedimensional half-space in the infinite depth case and $\mathbb{R}^{2} \times(-d, 0)$ in the case of constant finite depth $d$. Let $G: D \times D \rightarrow \overline{\mathbb{C}}$ be a Green function which satisfies the boundary value problem

$$
\begin{aligned}
\Delta_{\mathbf{x}} G(\mathbf{x} ; \xi) & =-\delta(\mathbf{x}-\xi), & \mathbf{x}, \xi & \in D, \\
\frac{\partial G}{\partial z} & =\alpha G, & & \text { at } z=0, \mathbf{x}, \xi \in D,
\end{aligned}
$$

as well as

$$
\sup _{\mathbf{x} \in D}\left|G(\mathbf{x} ; \xi)-\frac{1}{4 \pi\|\mathbf{x}\|}\right|<\infty, \quad \xi \in D
$$


in the case of infinite depth and

$$
\frac{\partial G}{\partial z}=0, \quad \text { at } z=-d, \mathbf{x}, \xi \in D
$$

in the case of constant finite depth $d$. A radiation condition, which may be expressed as

$$
\lim _{\bar{r} \rightarrow \infty} \sqrt{\bar{r}}\left(\frac{\partial}{\partial \bar{r}}-\mathrm{i} k\right) G(\mathbf{x} ; \xi)=0, \quad z<0, \xi \in D
$$

where $k$ is the wavenumber and $\bar{r}^{2}=x^{2}+y^{2}$, must also be fulfilled to ensure outgoing waves.

Denoting the immersed surface of the body with $\Gamma$, the integral equation for the scattered potential (the potential with the ambient wave subtracted) is then given by

$$
\frac{1}{2} \phi^{\mathrm{S}}(\mathbf{x})=\int_{\Gamma} \phi^{\mathrm{S}}(\xi) \frac{\partial G}{\partial n_{\xi}}(\mathbf{x} ; \xi)-G(\mathbf{x} ; \xi) \frac{\partial \phi^{\mathrm{S}}}{\partial n_{\xi}}(\xi) \mathrm{d} \sigma_{\xi}, \quad \mathbf{x} \in \Gamma .
$$

In many situations equation (2) can be further simplified by the utilisation of boundary conditions at the immersed surface. It is also possible to derive related integral equations, for instance by expanding the scattered potential as sources on the immersed surface.

Solutions for the free surface Green function given by equations (1) are well known and have been given in many forms. Since this paper is concerned with the Green function for infinite depth, the representations of the infinite depth Green function is considered. Before presenting these Green functions the following notation must be introduced. Let $(r, \theta, z)$ be cylindrical coordinates such that

$$
\begin{aligned}
(x, y, z) & \mapsto(r, \theta, z), \\
x-a & =r \cos \theta, \\
y-b & =r \sin \theta, \\
z & =z,
\end{aligned}
$$

and let $R_{0}$ and $R_{1}$ denote the distance from the source point $\xi=(a, b, c)$ and the distance from the mirror source point $\bar{\xi}=(a, b,-c)$ respectively, $R_{0}^{2}=(x-a)^{2}+(y-b)^{2}+(z-c)^{2}$ and $R_{1}^{2}=(x-a)^{2}+(y-b)^{2}+(z+c)^{2}$. In infinite depth, the Green function $G$, for $r>0$, was given by Havelock [5] as

$$
\begin{aligned}
G(\mathbf{x} ; \xi)= & \frac{\mathrm{i} \alpha}{2} e^{\alpha(z+c)} H_{0}^{(1)}(\alpha r)+\frac{1}{4 \pi R_{0}}+\frac{1}{4 \pi R_{1}} \\
& -\frac{1}{\pi^{2}} \int_{0}^{\infty} \frac{\alpha}{\eta^{2}+\alpha^{2}}(\alpha \cos \eta(z+c)-\eta \sin \eta(z+c)) K_{0}(\eta r) \mathrm{d} \eta,
\end{aligned}
$$


where $H_{0}^{(1)}$ and $K_{0}$ denote the Hankel function of the first kind and the modified Bessel function of the second kind, both of order zero as defined in Abramowitz \& Stegun [1]. This Green function is referred to as Havelock's Green function. It should be noted that Havelock's Green function can also be written in the following closely related form,

$$
\begin{aligned}
G(\mathbf{x} ; \xi)= & \frac{\mathrm{i} \alpha}{2} e^{\alpha(z+c)} H_{0}^{(1)}(\alpha r)+\frac{1}{4 \pi R_{0}} \\
& +\frac{1}{2 \pi^{2}} \int_{0}^{\infty} \frac{\left(\eta^{2}-\alpha^{2}\right) \cos \eta(z+c)+2 \eta \alpha \sin \eta(z+c)}{\eta^{2}+\alpha^{2}} K_{0}(\eta r) \mathrm{d} \eta
\end{aligned}
$$

[10]. An equivalent representation is due to Kim [9] for $r>0$, although implicitly given in the work of Havelock [5], and is given by

$$
\begin{aligned}
G(\mathbf{x} ; \xi)=\frac{1}{4 \pi R_{0}}+\frac{1}{4 \pi R_{1}}-\frac{\alpha}{4} e^{\alpha(z+c)}( & \mathbf{H}_{0}(\alpha r)+Y_{0}(\alpha r)-2 \mathrm{i} J_{0}(\alpha r) \\
& \left.+\frac{2}{\pi} \int_{z+c}^{0} \frac{\mathrm{e}^{-\alpha \eta}}{\sqrt{r^{2}+\eta^{2}}} \mathrm{~d} \eta\right)
\end{aligned}
$$

where $J_{0}$ and $Y_{0}$ are the Bessel functions of order zero of the first and second kind and $\mathbf{H}_{0}$ is the Struve function of order zero. This Green function is referred to as Kim's Green function.

The choice of best representation for the Green function is generally determined by numerical considerations, specifically which Green function can be calculated with the least number of floating point operations. As the Green function depends on a number of parameters the choice of best Green function can vary but it is generally thought that Kim's Green function is the best numerically [6] for a naive implementation based on numerical integration. However, it should be noted as stated in the introduction, for the most efficient numerical evaluation of the Green's functions the methods of Noblesse or Newman should be used.

The most important representation of the finite depth free surface Green function is the eigenfunction expansion given by John [7],

$$
\begin{aligned}
G(\mathbf{x} ; \xi)= & \frac{\mathrm{i}}{2} \frac{\alpha^{2}-k^{2}}{\left(\alpha^{2}-k^{2}\right) d-\alpha} \cosh k(z+d) \cosh k(c+d) H_{0}^{(1)}(k r) \\
& +\frac{1}{\pi} \sum_{m=1}^{\infty} \frac{k_{m}^{2}+\alpha^{2}}{\left(k_{m}^{2}+\alpha^{2}\right) d-\alpha} \cos k_{m}(z+d) \cos k_{m}(c+d) K_{0}\left(k_{m} r\right)
\end{aligned}
$$

valid for $r>0$. This is required in the derivation of our new representation for the infinite depth Green function. The positive wavenumber $k$ is related to the radian frequency $\omega$ by

$$
\alpha=k \tanh k d
$$


and the values of $k_{m}, m>0$, are given as positive real roots of the dispersion relation

$$
\alpha+k_{m} \tan k_{m} d=0 .
$$

This representation for the finite depth Green function is an eigenfunction expansion with the terms $\cosh k(z+d) H_{0}^{(1)}(k r)$ and $\cos k_{m}(z+d) K_{0}\left(k_{m} r\right)$ being the eigenfunctions as can be seen if separation of variables is applied in cylindrical coordinates. Such separation of variables is widely used, for example it is the basis of all the methods which exploit axisymmetry. Also, the eigenfunction expansion has the property that it is, roughly speaking, the best basis in which to represent the scattered potential. This property is used in a number of methods, most notably the scattering theory of Kagemoto $\mathcal{E}^{3}$ Yue [8]. Furthermore, the eigenfunction expansion of the Green function is required to represent the scattered potential in the cylindrical eigenfunctions.

It should be noted that the finite depth Green function is considerably more complicated than the infinite depth Green function and it is therefore always preferable to use the infinite depth representation if it is appropriate.

\section{Derivation of the eigenfunction representation of the infinite depth Green function}

In this section we derive a representation of the infinite depth Green function in the eigenfunction expansion of the potential for outgoing waves. In the case of infinite depth the decaying eigenvalues (which in finite depth are given by the discrete roots of equation (8)) are given as elements of a continuous line. This does not pose any problems to the eigenfunction expansion and simply requires that the sum is replaced by an integral. The eigenfunction expansion for the scattered potential is given by

$$
\begin{aligned}
\phi^{\mathrm{S}}(r, \theta, z)= & \mathrm{e}^{\alpha z} \sum_{\nu=-\infty}^{\infty} A_{\nu}^{0} H_{\nu}^{(1)}(\alpha r) \mathrm{e}^{\mathrm{i} \nu \theta} \\
& +\int_{0}^{\infty}\left(\cos \eta z+\frac{\alpha}{\eta} \sin \eta z\right) \sum_{\nu=-\infty}^{\infty} A_{\nu}(\eta) K_{\nu}(\eta r) \mathrm{e}^{\mathrm{i} \nu \theta} \mathrm{d} \eta,
\end{aligned}
$$

where the coefficients for the propagating modes $A_{\nu}^{0}$ are discrete and the coefficients for the decaying modes $A_{\nu}(\cdot)$ are functions. Havelock's representation for the Green function (3) and the representation given in equation (4) are similar to the eigenfunction expansion except for the $1 / R$-terms.

An eigenfunction representation for the infinite depth Green function is derived by letting the depth tend to infinity in the finite depth Green function (in the eigenfunction representation) (6). At first, the term which represents 
the propagating modes is considered,

$$
\frac{\mathrm{i}}{2} \frac{\alpha^{2}-k^{2}}{\left(\alpha^{2}-k^{2}\right) d-\alpha} \cosh k(z+d) \cosh k(c+d) H_{0}^{(1)}(k r) .
$$

The limit as the depth tends to infinity can be taken by making use of the dispersion relation (7), and the hyperbolic function relationships [1],

$$
\tanh k d=\frac{\alpha}{k} \quad \Rightarrow \quad \cosh k d= \pm \frac{k}{\sqrt{k^{2}-\alpha^{2}}}
$$

This result can be used to rewrite the hyperbolic cosines,

$$
\cosh k(z+d) \cosh k(c+d)=\frac{k^{2}}{k^{2}-\alpha^{2}} \frac{\cosh k(z+d)}{\cosh k d} \frac{\cosh k(c+d)}{\cosh k d} .
$$

Expanding the quotients,

$$
\frac{\cosh k(z+d)}{\cosh k d}=\cosh k z+\tanh k d \sinh k z,
$$

the term for the propagating modes (10) can be written as

$\frac{\mathrm{i}}{2} \frac{k^{2}}{\left(k^{2}-\alpha^{2}\right) d+\alpha}(\cosh k z+\tanh k d \sinh k z)(\cosh k c+\tanh k d \sinh k c) H_{0}^{(1)}(k r)$.

Noting that $\lim _{d \rightarrow \infty} \tanh (d)=1$ and keeping in mind the dispersion relation (7) as well as $\cosh z+\sinh z=\mathrm{e}^{z}$, the limit of the term for the propagating modes is given by

$$
\frac{\mathrm{i} \alpha}{2} \mathrm{e}^{\alpha(z+c)} H_{0}^{(1)}(\alpha r)
$$

Unsurprisingly, this is the same as the term for the propagating modes in equations (3) and (4) since these terms were already given in the eigenfunction representation.

Now the term for the decaying modes from equation (6),

$$
\frac{1}{\pi} \sum_{m=1}^{\infty} \frac{k_{m}^{2}+\alpha^{2}}{\left(k_{m}^{2}+\alpha^{2}\right) d-\alpha} \cos k_{m}(z+d) \cos k_{m}(c+d) K_{0}\left(k_{m} r\right),
$$

is considered. The cosine terms are expanded as

$$
\begin{aligned}
\cos k_{m}(z+d) \cos k_{m} & (c+d)= \\
& \cos k_{m} z \cos k_{m} c \cos ^{2} k_{m} d+\sin k_{m} z \sin k_{m} c \sin ^{2} k_{m} d \\
& -\left(\cos k_{m} z \sin k_{m} c+\cos k_{m} c \sin k_{m} z\right) \cos k_{m} d \sin k_{m} d
\end{aligned}
$$

and the dispersion relation (8) is used. Since

$$
\tan k_{m} d=-\frac{\alpha}{k_{m}}
$$


it follows from the trigonometric relations for the angles and sides of a triangle that the sine and cosine are given by

$$
\sin k_{m} d= \pm \frac{\alpha}{\sqrt{k_{m}^{2}+\alpha^{2}}} \quad \text { and } \quad \cos k_{m} d=\mp \frac{k_{m}}{\sqrt{k_{m}^{2}+\alpha^{2}}}
$$

Substituting this into the expansion of the cosine terms gives

$$
\begin{aligned}
\cos k_{m}(z+d) \cos k_{m}(c+d) & \\
= & \cos k_{m} z \cos k_{m} c \frac{k_{m}^{2}}{k_{m}^{2}+\alpha^{2}}+\sin k_{m} z \sin k_{m} c \frac{\alpha^{2}}{k_{m}^{2}+\alpha^{2}} \\
& +\left(\cos k_{m} z \sin k_{m} c+\cos k_{m} c \sin k_{m} z\right) \frac{\alpha k_{m}}{k_{m}^{2}+\alpha^{2}} \\
= & \frac{1}{2}\left(\cos k_{m}(z-c)+\frac{k_{m}^{2}-\alpha^{2}}{k_{m}^{2}+\alpha^{2}} \cos k_{m}(z+c)\right. \\
& \left.\quad+\frac{2 \alpha k_{m}}{k_{m}^{2}+\alpha^{2}} \sin k_{m}(z+c)\right) .
\end{aligned}
$$

Using the asymptotic expansion of $k_{m}, k_{m} \sim\left(m-\frac{1}{2}\right) \frac{\pi}{d}, m>0$, for large $\alpha d$ and writing $h=\pi / d$, the term for the decaying modes becomes

$$
\begin{aligned}
& \frac{1}{2 \pi^{2}} h \sum_{m \in M} \frac{k_{m}^{2}+\alpha^{2}}{\left(k_{m}^{2}+\alpha^{2}\right)-\frac{\alpha}{d}}\left(\cos m h(z-c)+\frac{(m h)^{2}-\alpha^{2}}{(m h)^{2}+\alpha^{2}} \cos m h(z+c)\right. \\
& \left.+\frac{2 \alpha m h}{(m h)^{2}+\alpha^{2}} \sin m h(z+c)\right) K_{0}(m h r),
\end{aligned}
$$

where $M$ is the set of odd multiples of $1 / 2, M:=\left\{m-1 / 2 \mid m \in \mathbb{N}_{>0}\right\}$. This is a Riemann sum and therefore the limit as $h$ tends to zero (which is equivalent to $d$ tending to infinity) is

$$
\frac{1}{2 \pi^{2}} \int_{0}^{\infty}\left(\cos \eta(z-c)+\frac{\eta^{2}-\alpha^{2}}{\eta^{2}+\alpha^{2}} \cos \eta(z+c)+\frac{2 \alpha \eta}{\eta^{2}+\alpha^{2}} \sin \eta(z+c)\right) K_{0}(\eta r) \mathrm{d} \eta .
$$

The Green function in infinite depth is therefore given by

$$
\begin{aligned}
G(\mathbf{x} ; \xi)= & \frac{\mathrm{i} \alpha}{2} \mathrm{e}^{\alpha(z+c)} H_{0}^{(1)}(\alpha r)+\frac{1}{2 \pi^{2}} \int_{0}^{\infty}(\cos \eta(z-c) \\
& \left.+\frac{\eta^{2}-\alpha^{2}}{\eta^{2}+\alpha^{2}} \cos \eta(z+c)+\frac{2 \alpha \eta}{\eta^{2}+\alpha^{2}} \sin \eta(z+c)\right) K_{0}(\eta r) \mathrm{d} \eta
\end{aligned}
$$

However, the objective was to represent the Green function in the eigenfunction expansion given in equation (9). For this reason, the terms depending on $z$ and $c$ have to be written as a product of each other. This can be accomplished 
by the following calculation,

$$
\begin{aligned}
\cos \eta(z-c) \frac{\eta^{2}-\alpha^{2}}{\eta^{2}+\alpha^{2}} \cos \eta(z+c)+\frac{2 \alpha \eta}{\eta^{2}+\alpha^{2}} \sin \eta(z+c) \\
=\cos \eta z\left(\cos \eta c+\frac{\eta^{2}-\alpha^{2}}{\eta^{2}+\alpha^{2}} \cos \eta c+\frac{2 \alpha \eta}{\eta^{2}+\alpha^{2}} \sin \eta c\right) \\
\quad+\sin \eta z\left(\sin \eta c-\frac{\eta^{2}-\alpha^{2}}{\eta^{2}+\alpha^{2}} \sin \eta c+\frac{2 \alpha \eta}{\eta^{2}+\alpha^{2}} \cos \eta c\right) \\
=\left(\frac{2 \eta^{2}}{\eta^{2}+\alpha^{2}} \cos \eta c+\frac{2 \alpha \eta}{\eta^{2}+\alpha^{2}} \sin \eta c\right) \\
\quad \quad \quad\left(\cos \eta z+\frac{2 \alpha^{2} \sin \eta c+2 \alpha \eta \cos \eta c}{2 \eta^{2} \cos \eta c+2 \alpha \eta \sin \eta c} \sin \eta z\right) \\
=2\left(\frac{\eta^{2}}{\eta^{2}+\alpha^{2}} \cos \eta c+\frac{\alpha \eta}{\eta^{2}+\alpha^{2}} \sin \eta c\right)\left(\cos \eta z+\frac{\alpha}{\eta} \sin \eta z\right) .
\end{aligned}
$$

The eigenfunction expansion of the three-dimensional free surface Green function is therefore given by

$$
\begin{aligned}
G(\mathbf{x} ; \xi)=\frac{\mathrm{i} \alpha}{2} \mathrm{e}^{\alpha(z+c)} H_{0}^{(1)}(\alpha r)+ & \frac{1}{\pi^{2}} \int_{0}^{\infty}\left(\cos \eta z+\frac{\alpha}{\eta} \sin \eta z\right) \\
& \times \frac{\eta^{2}}{\eta^{2}+\alpha^{2}}\left(\cos \eta c+\frac{\alpha}{\eta} \sin \eta c\right) K_{0}(\eta r) \mathrm{d} \eta
\end{aligned}
$$

\section{Numerical calculation of the infinite depth Green function}

The eigenfunction expansion of the Green function was derived for theoretical purposes but it also has some numerical advantages over the existing representations. In this section the numerical properties of the Green function in eigenfunction representation are investigated and comparisons are made to the existing representations of the Green function. These comparisons are only for a naive implementation in which the integrals are directly calculated.

Examining the representations of the Green function given by equations (3), (4), (5) and (12) it can be seen that the numerically difficult parts of the Green 
functions are given by the following terms

$$
\begin{aligned}
& \int_{0}^{\infty} \frac{\alpha^{2} \cos \eta(z+c)-\eta \alpha \sin \eta(z+c)}{\eta^{2}+\alpha^{2}} K_{0}(\eta r) \mathrm{d} \eta, \\
& \int_{0}^{\infty} \frac{\left(\eta^{2}-\alpha^{2}\right) \cos \eta(z+c)+2 \eta \alpha \sin \eta(z+c)}{\eta^{2}+\alpha^{2}} K_{0}(\eta r) \mathrm{d} \eta, \\
& \int_{z+c}^{0} \frac{\mathrm{e}^{-\alpha \eta}}{\sqrt{r^{2}+\eta^{2}}} \mathrm{~d} \eta \\
& \int_{0}^{\infty}\left(\cos \eta z+\frac{\alpha}{\eta} \sin \eta z\right) \frac{\eta^{2}}{\eta^{2}+\alpha^{2}}\left(\cos \eta c+\frac{\alpha}{\eta} \sin \eta c\right) K_{0}(\eta r) \mathrm{d} \eta .
\end{aligned}
$$

The other parts of the Green functions can be evaluated easily and are available in most modern software packages, with the exception of the Struve function which appears in Kim's representation. Although the difficulty in the Struve function is ignored in the following comparison, it should be noted that the presence of this function imposes programming difficulties, since the Struve function is not standard, as well as requiring numerical effort.

The disadvantage of the first two representations is that the integrand is singular at zero. The numerical evaluation of the integrals is therefore rather expensive. Hearn [6] showed that the evaluation of Kim's Green function (the numerically difficult part of which is given by equation $(13 \mathrm{c})$ ) is in general much easier than that of Havelock's Green function (the numerically difficult part of which is given by equation (13a) or equation (13b)) since it only involves the integration of a non-singular integrand and the evaluation of the Struve function. This numerical advantage was especially true in common applications such as ship-wave calculations.

The eigenfunction representation of the Green function does not involve any uncommon special function and the integrand of the numerically difficult term (equation (13d)) is non-singular over the integration interval. While the interval of integration is infinite, the fast decay of the modified Bessel function of the second kind causes the integrand to decay quickly for large argument and the integral therefore only needs to be calculated for a small interval.

Figure 1 shows the integrands of the different terms in equations (13). In all plots, the remaining variables are chosen to be $\alpha=\pi, c=-1, z=-1$ and $r=1$. Plots (c) and (d), which correspond to Kim's representation and the eigenfunction expansion, are numerically much easier to integrate because they do not have a singularity at zero.

Since Kim's representation is numerically much better than Havelock's representation, the eigenfunction expansion is numerically compared to Kim's representation more thoroughly. At first, a few qualitative remarks comparing 
(a)

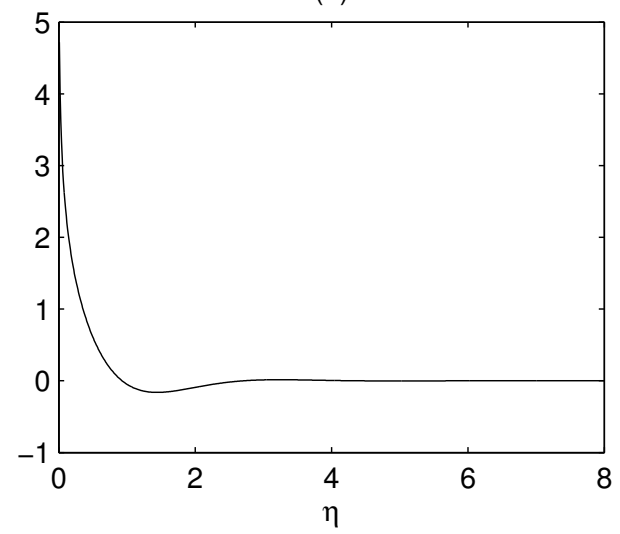

(c)

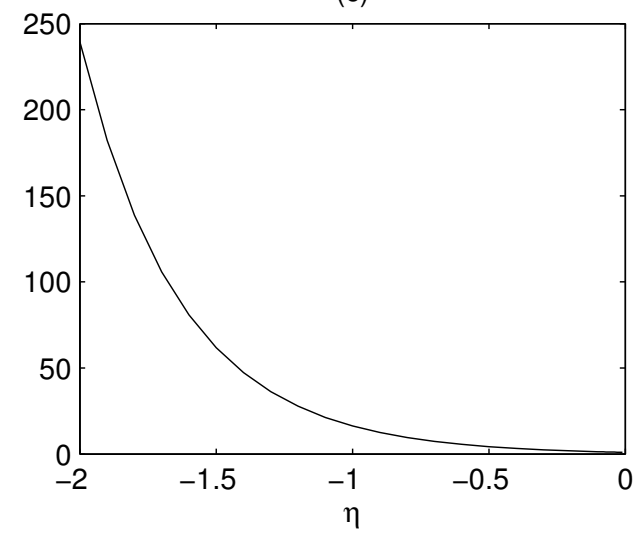

(b)

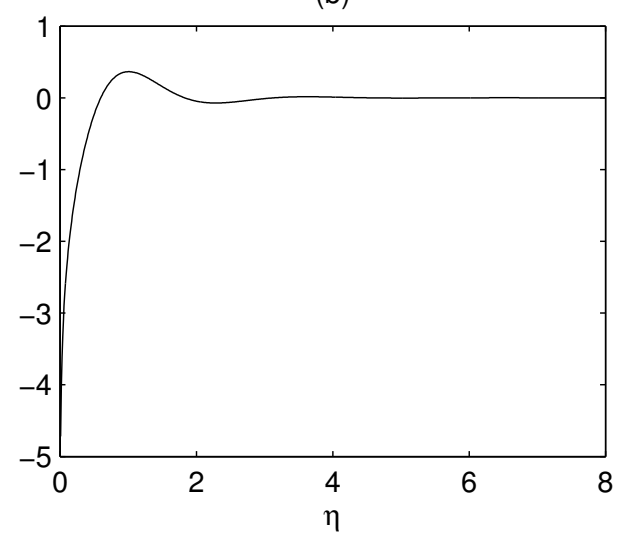

(d)

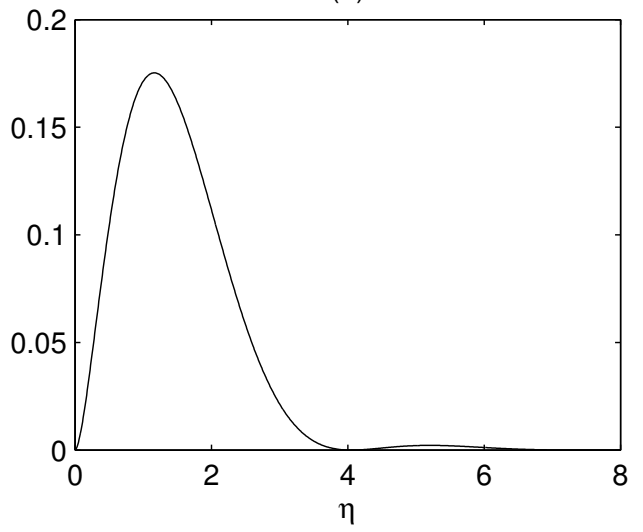

Fig. 1. The graphs of the integrands in equations (13) for $\alpha=\pi, c=-1, z=-1$ and $r=1$. Plot (a) corresponds to equation (13a) etc.

the two methods are made before quantitative results are presented. As can be seen from equations (13c) and (13d), interchanging the values of $z$ and $c$ makes no difference in the calculation. If the absolute values of $z$ and $c$ become large, the integrand of the eigenfunction representation starts to oscillate more while the integration interval in Kim's representation becomes larger which results in the need to integrate a steeper function (since $z, c \leq 0$ always). An increase in the radial coordinate, $r$, smoothes the integrand in the eigenfunction representation since it accelerates the decay of the Bessel function but barely affects the integration in Kim's representation. A change in the wavenumber (since $\alpha=k$ for infinite depth) barely changes the integrand in the eigenfunction representation but dramatically alters the integrand in Kim's representation because it changes its steepness.

Table 1 compares the required function evaluations when integrating the integrands from Kim's representation in equation (13c) and the eigenfunction representation in equation (13d) with the adaptive Simpson quadrature provided by MatLaB. The interval of integration in the eigenfunction representation is from zero until the point at which the integrand is strictly less than $10^{-6}$. For the cases illustrated in table 1 this upper value for the interval of integration 


\begin{tabular}{|c|c|c|c|c|}
\hline Tolerance & \multicolumn{2}{|c|}{ Kim's repr. eigenfunction repr. } & \multicolumn{2}{|c|}{ Kim's repr. eigenfunction repr } \\
\hline & \multicolumn{2}{|c|}{$\alpha=\pi, c=-1, z=-1, r=1$} & \multicolumn{2}{|c|}{$\alpha=\pi, c=-1, z=-1 / 2, r=1$} \\
\hline $10^{-2}$ & 13 & 5 & 9 & 5 \\
\hline $10^{-4}$ & 37 & 25 & 25 & 21 \\
\hline \multirow[t]{2}{*}{$10^{-6}$} & 89 & 61 & 53 & 53 \\
\hline & \multicolumn{2}{|c|}{$\alpha=\pi, c=-1, z=-1, r=1 / 2$} & \multicolumn{2}{|c|}{$\alpha=\pi, c=-1, z=-1, r=2$} \\
\hline $10^{-2}$ & 13 & 17 & 13 & 5 \\
\hline $10^{-4}$ & 41 & 37 & 33 & 17 \\
\hline \multirow[t]{2}{*}{$10^{-6}$} & 93 & 117 & 89 & 41 \\
\hline & \multicolumn{2}{|c|}{$\alpha=\pi / 2, c=-1, z=-1, r=1$} & \multicolumn{2}{|c|}{$\alpha=2 \pi, c=-1, z=-1, r=1$} \\
\hline $10^{-2}$ & 5 & 5 & 57 & 5 \\
\hline $10^{-4}$ & 17 & 21 & 133 & 17 \\
\hline $10^{-6}$ & 33 & 65 & 365 & 73 \\
\hline
\end{tabular}

Table 1

The number of function evaluations when numerically integrating the integrands from Kim's representation (equation (13c)) and the eigenfunction expansion (equation (13d)).

is between 6 and 26 .

The results in table 1 show that the number of function evaluations required by Kim's representation is generally greater, often by a considerable amount, than the number required by the eigenfunction representation. In the only cases where Kim's representation performs better $(\alpha=\pi / 2, c=-1, z=$ $-1, r=1$ and $\alpha=\pi, c=-1, z=-1, r=1 / 2)$ it only does so by a small amount. Of course these results do not form a definitive test that the eigenfunction representation is better since ways to optimise the integration have not been investigated or the different costs in evaluating the integrands have not been taken into account. However, these results, especially because the cost of evaluating the Struve function which Kim's representation requires has not been considered, do indicate that the eigenfunction representation does perform well numerically in comparison to Kim's representation.

\section{The eigenfunction representation of the Green function in a co- ordinate system with a different origin}

As explained previously, a representation of the infinite depth Green function as an expansion in eigenfunctions has been obtained. In this expansion the 
origin of the circular coordinate systems was at the water surface above the source point. In most cases this does not pose any problems, but in some applications, for example in the calculation of diffraction transfer matrices as required in the application of Kagemoto \& Yue's interaction theory [8] for bodies of arbitrary shapes, it is necessary to represent the potential in terms of a coordinate system with a fixed origin. This can be accomplished using Graf's addition theorem for Bessel and Hankel functions. In finite depth, this has been done by Black [2] and Fenton [3] and used for Kagemoto $\& 5$ Yue's interaction theory by Goo $\&$ Yoshida [4]. In infinite depth however, this representation of the Green function in an eigenfunction expansion with fixed origin has not been possible because of the lack of a representation of the Green function in the eigenfunction expansion with the coordinate system centred at the water surface above the source point. Using the previously derived Green function, the eigenfunction representation for a fixed coordinate system is developed here.

From now on, the cylindrical coordinate system $(r, \theta, z)$ is assumed fixed at some point of the undisturbed water surface. The potential due to a source point $\mathbf{s}$ whose coordinates in the cylindrical coordinate system are $(s, \varphi, c)$ is to be represented at a point $\mathbf{r}$ whose coordinates are $(r, \theta, z)$. So far only an eigenfunction representation of the potential in a coordinate system centred at $\mathbf{s}$ has been obtained. Figure 2 shows a two-dimensional view of this setting with the $z$-dependence not shown.

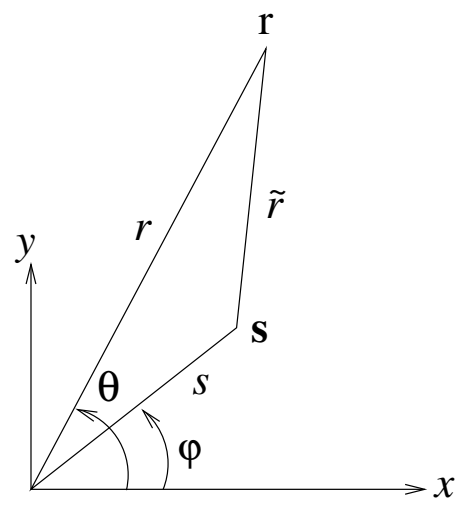

Fig. 2. Plan view of the coordinate systems with a point source at $\mathbf{s}=(s, \varphi, c)$.

The basis of the transformation is Graf's addition theorem for Bessel functions which is given in Abramowitz \& Stegun [1]. It can be applied to yield the following identities

$$
\begin{aligned}
H_{0}^{(1)}(\alpha \tilde{r}) & =\sum_{\nu=-\infty}^{\infty} H_{\nu}^{(1)}(\alpha r) J_{\nu}(\alpha s) \mathrm{e}^{\mathrm{i} \nu(\theta-\varphi)} \\
K_{0}(\eta \tilde{r}) & =\sum_{\nu=-\infty}^{\infty} K_{\nu}(\eta r) I_{\nu}(\eta s) \mathrm{e}^{\mathrm{i} \nu(\theta-\varphi)}
\end{aligned}
$$


for $r>s$ and

$$
\begin{aligned}
H_{0}^{(1)}(\alpha \tilde{r}) & =\sum_{\nu=-\infty}^{\infty} H_{\nu}^{(1)}(\alpha s) J_{\nu}(\alpha r) \mathrm{e}^{\mathrm{i} \nu(\theta-\varphi)} \\
K_{0}(\eta \tilde{r}) & =\sum_{\nu=-\infty}^{\infty} K_{\nu}(\eta s) I_{\nu}(\eta r) \mathrm{e}^{\mathrm{i} \nu(\theta-\varphi)}
\end{aligned}
$$

for $s>r$ where $I_{\nu}$ is the modified Bessel function of the first kind of order $\nu$.

The application of equations (14) to the eigenfunction representation of the Green function, equation (12), gives the potential due to a source point at $\mathbf{s}=(s, \varphi, c)$,

$$
\begin{aligned}
& G(r, \theta, z ; s, \varphi, c)= \\
& \quad \frac{\mathrm{i} \alpha}{2} \mathrm{e}^{\alpha(z+c)} \sum_{\nu=-\infty}^{\infty}\left\{\begin{array}{l}
H_{\nu}^{(1)}(\alpha r) J_{\nu}(\alpha s) \\
H_{\nu}^{(1)}(\alpha s) J_{\nu}(\alpha r)
\end{array}\right\} \mathrm{e}^{\mathrm{i} \nu(\theta-\varphi)}+\frac{1}{\pi^{2}} \int_{0}^{\infty}\left(\cos \eta z+\frac{\alpha}{\eta} \sin \eta z\right) \\
& \quad \times \frac{\eta^{2}}{\eta^{2}+\alpha^{2}}\left(\cos \eta c+\frac{\alpha}{\eta} \sin \eta c\right) \sum_{\nu=-\infty}^{\infty}\left\{\begin{array}{l}
K_{\nu}(\eta r) I_{\nu}(\eta s) \\
K_{\nu}(\eta s) I_{\nu}(\eta r)
\end{array}\right\} \mathrm{e}^{\mathrm{i} \nu(\theta-\varphi)} \mathrm{d} \eta .
\end{aligned}
$$

The upper terms in the curly brackets need to be used when $r>s$, the lower ones when $s>r$. For $s>r$ the source potential is therefore given in the eigenfunction expansion for incoming waves, for $r>s$ it is given in the eigenfunction expansion for outgoing waves. The expansion for outgoing waves is applied in scattering or radiation problems where the coordinate system must be centred at the mean centre position of the body.

\section{Summary}

A representation of the free surface Green function for infinite depth in the eigenfunctions of an outgoing wave has been derived. The new representation of the Green function has a number of advantages over existing representations. It does not involve any uncommon special functions or the integration of a singular integrand. The infinite interval of integration does not cause difficulties because of the fast decay of the integrand. For a naive implementation in which the integral is calculated directly the new representation of the Green function is found to compare favourably to the representation due to Kim. The new representation of the Green function in eigenfunctions can also be transformed to a coordinate system which is not centred at the source point of the Green function while still retaining the eigenfunction expansion. This transformation is required in certain applications, for example in the calculation of the diffraction transfer matrices for bodies of arbitrary shape. 


\section{Notation}

$\Gamma \quad$ the immersed surface of a floating or submerged body

$D$ the lower three-dimensional half-space in the infinite depth case and $\mathbb{R}^{2} \times(-d, 0)$ in the finite depth case

$\mathbf{x}$ a point in the water, $\mathbf{x}=(x, y, z)$ (Cartesian coordinates)

$\mathbf{r}$ a point in the water, $\mathbf{r}=(r, \theta, z)$ (cylindrical coordinates)

$\xi \quad$ a point source in the water, $\xi=(a, b, c)$ (Cartesian coordinates)

$\mathbf{s} \quad$ a point source in the water, $\mathbf{s}=(s, \varphi, c)$ (cylindrical coordinates)

$\phi^{\mathrm{S}} \quad$ the time-independent scattered water velocity potential, $\Phi^{\mathrm{S}}(x, y, z, t)=\operatorname{Re}\left\{\phi^{\mathrm{S}}(x, y, z) e^{-\mathrm{i} \omega t}\right\}$

$G$ a Green function

$J_{\nu} \quad$ Bessel function of the first kind and of integer order $\nu$

$Y_{\nu} \quad$ Bessel function of the second kind and of integer order $\nu$

$I_{\nu} \quad$ modified Bessel function of the first kind and of integer order $\nu$

$K_{\nu} \quad$ modified Bessel function of the second kind and of integer order $\nu$

$H_{\nu}^{(1)} \quad$ Hankel function of the first kind and of integer order $\nu$

$\mathbf{H}_{0} \quad$ Struve function of order zero

i the imaginary unit, $\mathrm{i}=\sqrt{-1}$

d the water depth

$g$ the acceleration due to gravity

$\omega \quad$ the radian frequency

$k \quad$ the wavenumber

$\alpha \quad \alpha=\omega^{2} / g$

$k_{m} \quad$ roots of the dispersion relation, equation (8) 


$$
\begin{aligned}
& A_{\nu}^{0} \quad \text { coefficients of the propagating modes of the water velocity } \\
& \text { potential in eigenfunction expansion } \\
& A_{\nu}(\cdot) \text { coefficient functions of the decaying modes of the water velocity } \\
& \text { potential in eigenmode expansion }
\end{aligned}
$$

\section{Acknowledgements}

Thanks to Winston Sweatman for his comments on the manuscript. This reseach was supported by Marsden Grant UOO004 and the German National Scholarship Foundation.

\section{References}

[1] M. Abramowitz and I. A. Stegun. Handbook of Mathematical Functions. Dover Publications (1964).

[2] J. L. Black. Wave forces on vertical axisymmetric bodies. J. Fluid Mech. 67, 369-376 (1975).

[3] J. D. Fenton. Wave forces on vertical bodies of revolution. J. Fluid Mech. 85 (2), 241-255 (1978).

[4] J.-S. Goo and K. Yoshida. A numerical method for huge semisubmersible responses in waves. SNAME Transactions 98, 365-387 (1990).

[5] T. H. Havelock. Waves due to a floating sphere making periodic heaving oscillations. Proc. Roy. Soc. Lond. A 231, 1-7 (1955).

[6] G. E. Hearn. Alternative methods of evaluating Green's function in threedimensional ship wave problems. J. Ship Res. 21, 89-93 (1977).

[7] F. John. On the motion of floating bodies II. Comm. Pure Appl. Math. 3, 45-101 (1950).

[8] H. Kagemoto and D. K. P. Yue. Interactions among multiple three-dimensional bodies in water waves: an exact algebraic method. J. Fluid Mech. 166, 189-209 (1986).

[9] W. D. Kim. On the harmonic oscillations of a rigid body on a free surface. $J$. Fluid Mech. 21, 427-451 (1965).

[10] C. M. Linton and P. McIver. Handbook of Mathematical Techniques for Wave / Structure Interactions. Chapman \& Hall /CRC (2001). 
[11] J. N. Newman. Algorithms for the free-surface Green function. J. Engg. Math. 19, 57-67 (1985).

[12] F. Noblesse. The Green function in the theory of radiation and diffraction of regular water waves by a body. J. Engg. Math. 16, 138-169 (1982).

[13] J. G. Telste and F. Noblesse. Numerical evaluations of the Green function of water-wave radiation and diffraction. J. Ship Res. 30 (2), 69-84 (1986). 